\title{
Atividade estrogênica de desreguladores endócrinos em águas superficiais do município de Santa Maria Madalena, Sudeste do Brasil
}

\author{
Estrogenic activity of endocrine disruptors in the surface \\ water of Santa Maria Madalena, Southeastern Brazil
}

\begin{abstract}
Danieli Lima da Cunha" ${ }^{*}$, Samuel Muylaert ${ }^{2}$, Marilia Teresa Lima do Nascimento', Giselle Gomes Moreira da Silva ${ }^{3} \odot$, Estefan Monteiro da Fonseca ${ }^{1} \odot$, Daniele Maia Bila ${ }^{3} \odot$, Paula de Novaes Sarcinelli ${ }^{4} \stackrel{(\infty)}{ }$, Ariane Leites Larentis ${ }^{4}$ ()
\end{abstract}

\begin{abstract}
RESUMO
Nas últimas décadas se intensificou o lançamento, no meio hídrico, de contaminantes com capacidade de desregulação endócrina. Nesse contexto, a aplicação e o aprimoramento de métodos analíticos eficientes passam a ser cada vez mais requeridos para verificar os potenciais impactos dessas substâncias sobre os organismos a elas expostos. Bioensaios podem ser conduzidos para esse fim, como, por exemplo, o ensaio in vitro yeast estrogen screen (YES), que permite a detecção de compostos estrogênicos e citotóxicos. Este estudo, aplicando tal ensaio, investigou a atividade estrogênica das águas e seus potenciais riscos à biota aquática de dois corpos hídricos do município de Santa Maria Madalena, no estado do Rio de Janeiro, Sudeste do Brasil. Observou-se atividade estrogênica apenas no córrego São Domingos, com concentrações de equivalente estradiol (EQ-E2) de 23 e 10.4 ng.L.-1 no período de estiagem e chuvoso, respectivamente. Os potenciais riscos desse nível de atividade estrogênica foram mensurados por meio de uma avaliação de risco, que revelou alto e médio risco nas estações seca e chuvosa, respectivamente. Cabe mencionar que, entre os corpos hídricos estudados, a área do córrego São Domingos é aquela com maior adensamento populacional e menor cobertura de coleta e tratamento de esgotos. O ensaio YES mostrou ser uma boa ferramenta para a detecção da presença de desreguladores endócrinos estrogênicos, que pode subsidiar o avanço do conhecimento de corpos hídricos e a formulação de legislação e regulamentações da área ambiental.
\end{abstract}

Palavras-chave: atividade estrogênica; desregulares endócrinos; estrogênios; águas superficiais; efluentes domésticos

\begin{abstract}
In recent decades, the release of contaminants with endocrine disrupting capacity into the water environment has intensified. In this context, the application and improvement of efficient analytical methods are increasingly required to verify the potential impacts of these substances on the organisms exposed to them. Bioassays can be conducted with this purpose, such as the yeast estrogen screen (YES) in vitro assay, which allows the detection of estrogenic and cytotoxic compounds. This study, applying the YES assay, investigated the estrogenic activity and its potential risks to the aquatic biota of two streams in Santa Maria Madalena, Rio de Janeiro State, Southeastern Brazil. Estrogenic activity was observed only in São Domingos stream, with estradiol equivalent (EEQ) concentrations of 23 and 10.4 ng. $\mathrm{L}^{-1}$ during the dry and rainy season, respectively. The potential risks of this level of estrogenic activity were evaluated by a risk assessment, which revealed a high and medium risk to the local biota in the dry and rainy seasons, respectively. Among the studied water bodies, São Domingos watershed has a bigger population density and less sewage collection and treatment comparatively to Ribeirão Santíssimo stream. The YES assay presented itself as a useful tool for spotting estrogenic endocrine disruptors, with the potential to help in expanding knowledge about water bodies and to create and enforce environmental legislation and regulations
\end{abstract}

Keywords: estrogenic activity; endocrine disruptors; estrogens; surface water; domestic sewage.

'Universidade Federal Fluminense - Niterói (RJ), Brasil.

Instituto Estadual do Ambiente - Rio de Janeiro (RJ), Brasil.

3Universidade do Estado do Rio de Janeiro - Rio de Janeiro (RJ), Brasil.

4Fundação Oswaldo Cruz - Rio de Janeiro (RJ). Brasil

*Autora correspondente: danielicunha@hotmail.com

Conflitos de interesse: os autores declaram não haver conflito de interesses.

Financiamento: nenhum.

Recebido: 14/09/2018 - Aceito: 20/09/2019 - Reg. ABES: 20180133 


\section{INTRODUÇÃO}

Dada a grande relevância dos desreguladores endócrinos, métodos têm sido desenvolvidos visando à sua determinação nas mais variadas matrizes (água superficial, sedimentos, material particulado suspenso, efluente de estação de tratamento de esgoto, esgoto bruto etc.) (WEN et al., 2018; WU et al., 2017; CUNHA et al., 2017; NIE et al., 2015). Esses novos microcontaminantes podem causar efeitos adversos em ecossistemas aquáticos, principalmente em espécies sensíveis, como peixes e anfíbios (LIU et al., 2018; NEGINTAJI et al., 2018; PORSERYD et al., 2017; VALCARCE et al., 2017; ABDEL-KHALEK, 2018; CUNHA et al., 2016; LI; CHEN; QIN, 2016; TAMSCHICK et al., 2016; GILBERT, 2012). As amostras ambientais são em sua maioria complexas e as substâncias de interesse estão normalmente em concentrações na ordem de $\mu \mathrm{g} . \mathrm{L}^{-1}$ e ng. $\mathrm{L}^{-1}$, tornando necessário o desenvolvimento de técnicas eficientes de análise (GLINEUR et al., 2018; CHORMEY et al., 2017; FERRERA; SANTANA; RODRÍGUEZ, 2013; GE et al., 2010).

Entre as técnicas instrumentais disponíveis para a identificação e quantificação de desreguladores endócrinos em amostras ambientais, a análise química é a mais utilizada, principalmente por meio da cromatografia. Mas, quando se objetiva mensurar os efeitos decorrentes de dada exposição ambiental a uma substância específica ou mistura de substâncias, há uma variedade de bioensaios in vitro que pode ser conduzida, como, por exemplo, os ensaios de transativação (KUNZ et al., 2017; DVOŘÁKOVÁ et al., 2016). Tais bioensaios são baseados em mecanismos de ação que induzem respostas pela estimulação de receptores hormonais e, posteriormente, com o aumento da expressão de um gene repórter. A resposta é observada pela alteração de cor ou pelo aumento da luminescência, que indica a presença da atividade estrogênica da substância ou amostra ambiental (KUNZ et al., 2015).

Desenvolvido por Routledge e Sumpter em 1996, o ensaio Yeast Estrogen Screen (YES) é um método de análise in vitro de transativação que vem sendo largamente empregado pela sua rapidez, reprodutibilidade e sensibilidade (ROUTLEDGE; SUMPTER, 1996). Esse bioensaio permite estimar a atividade estrogênica total de uma mistura de substâncias (FANG et al., 2016; ALVAREZ et al., 2013; BRIX et al., 2010) e utiliza a levedura Saccharomyces cerevisiae geneticamente modificada, que possui uma sequência de DNA de receptor de estrogênio humano (REh) no seu genoma, como mostra a Figura 1. Quando há a presença de alguma substância estrogênica, esta interage com o REh, causando a expressão do gene receptor Lac-Z, que por sua vez produz a enzima $\beta$-galactosidase. Essa enzima é excretada no meio, metabolizando o substrato cromogênico (chlorophenol red- $\beta$-D-galactopyranoside - CPRG). A resposta colorimétrica desse bioensaio é então mensurada por espectrofotometria.

Por fim, os resultados obtidos são comparados a um padrão (estradiol E2), o que possibilita um resultado numérico em termos do equivalente estradiol (EQ-E2) (ROUTLEDGE; SUMPTER, 1996). Um dos principais motivos para adotar o E2 como padrão e não outros estrogênios, por exemplo o $17 \alpha$-etinilestradiol (EE2) ou estriol (E1), é que o E2 tem uma potência in vitro e in vivo entre EE2 e E1. Portanto, é possível avaliar os efeitos de uma mistura de forma mais precisa, uma vez que as amostras ambientais podem conter mais de um tipo de estrogênio (KUNZ et al., 2015).

O EQ-E2, utilizado como unidade universal para medir a atividade cumulativa das substâncias estrogênicas presentes na amostra, é ainda empregado na avaliação de risco. Seguindo essa metodologia, podem-se comparar tais resultados com padrões de referência, como, por exemplo, o padrão médio da qualidade ambiental (annual average environmental quality standards - AA EQS) da Diretiva-Quadro da Água da União Europeia (Water Framework Directive WFD) que, com base em dados de efeitos ecotoxicológicos, adotou a concentração limite de 0.4 ng.L.-1 para o E2 - nível de efeito adverso não observado para esse estrogênio (CUNHA et al., 2016; KUNZ et al., 2015; SCHER, 2011).

A Comissão do Meio Ambiente do Parlamento Europeu, com a Diretiva 2013/39/EU, incluiu o E2 na primeira lista de vigilância da WFD. Segundo a Diretiva, o uso de ferramentas baseadas em efeitos, como o ensaio YES, pode ajudar na elaboração e implementação de programas de monitoramento e fornecer um vínculo entre avaliações químicas e ecológicas, ajudando assim na avaliação da qualidade da água (CUNHA et al., 2016; JRC, 2015; UNIÃO EUROPEIA, 2013).

Diante da crescente preocupação com a qualidade da água e a fim de minimizar os riscos causados pela poluição hídrica, países têm investido no estudo da contaminação de vários compostos no meio ambiente. Nesse contexto, o presente estudo objetiva analisar a presença de estrogênios e seus potenciais efeitos adversos à biota aquática em dois corpos hídricos do município de Santa Maria Madalena. Com essa finalidade, analisou-se a atividade estrogênica das amostras pelo ensaio YES e realizou-se a avaliação de risco, de acordo com a metodologia proposta por KUNZ et al. (2015).

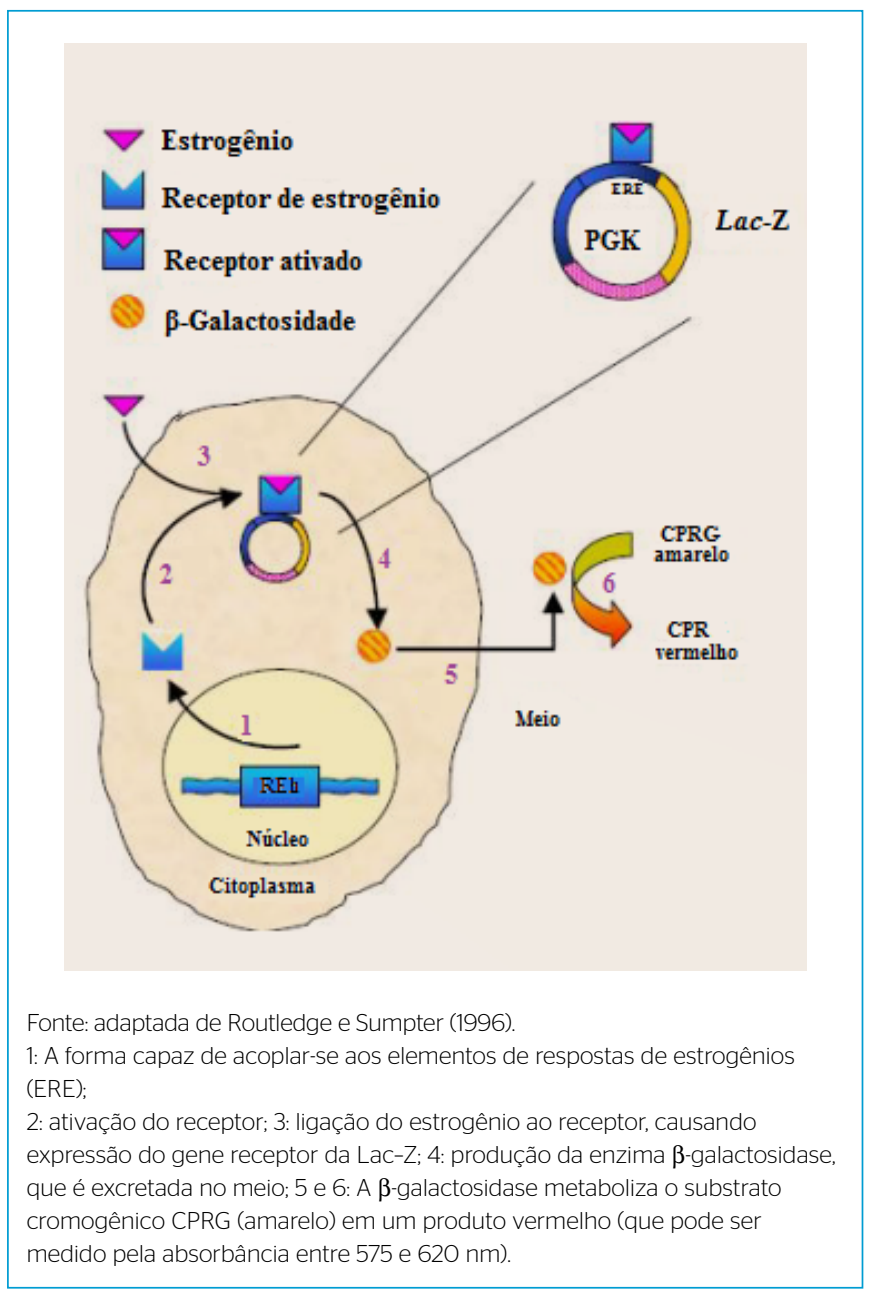

Figura 1 - Esquema de expressão da atividade estrogênica pela levedura Saccharomyces cerevisiae recombinante. 


\section{METODOLOGIA}

\section{Área de estudo}

O município de Santa Maria Madalena está localizado na região serrana do estado do Rio de Janeiro, no Sudeste do Brasil (Figura 2). Esse município, principalmente a sua área urbana, foi escolhido para o desenvolvimento deste trabalho por possuir corpos hídricos que nascem na própria área de estudo, não tendo, portanto, influência de outros municípios vizinhos.

Além disso, por ser uma região que não possui indústrias, mineradoras, agricultura, pecuária etc., pode-se considerar que a ocorrência de desreguladores endócrinos nos corpos hídricos locais está diretamente relacionada aos efluentes sanitários não tratados lançados diretamente nesses córregos, ou também aos efluentes de estações de tratamento de esgoto (ETE), uma vez que a literatura mostra que as ETE não eliminam eficientemente esse tipo de poluentes (CUNHA et al., 2017; BRANDT et al., 2013; AQUINO; BRANDT; CHERNICHARO, 2013).

Com relação ao esgotamento sanitário, a área urbana de Santa Maria Madalena possui duas ETE, e ambas aplicam um sistema de tratamento biológico de nível secundário. Conforme indicado no mapa da Figura 2, a ETE
I está instalada próximo ao córrego São Domingos e recebe contribuição de um único bairro (Cidade Alta), enquanto a ETE II está localizada no córrego Ribeirão Santíssimo e recebe esgoto bruto de dois bairros (Largo do Machado e Arranchadouro). Todos os outros bairros não são atendidos por qualquer ETE (PMSB, 2012), de modo que boa parte dos efluentes sanitários da área estudada não recebe nenhum tratamento antes de ser descarregada em corpos de água.

\section{Amostragem}

A coleta das amostras de água superficial foi realizada nos dois principais corpos hídricos que drenam a cidade: córrego São Domingos (2156'29,87"S

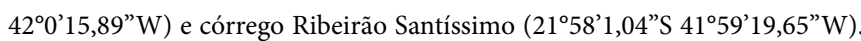
Esses pontos estão localizados a jusante de todas as contribuições da área urbana do $1^{\circ}$ distrito de Santa Maria Madalena (Figura 2).

Foram realizadas duas campanhas amostrais, a primeira em agosto de 2014 (fim do período da estiagem) e a segunda em novembro de 2014 (início do período chuvoso).

A amostragem foi do tipo composta, que consiste na combinação de amostras individuais de água (com o mesmo volume), tomadas em intervalos de tempo

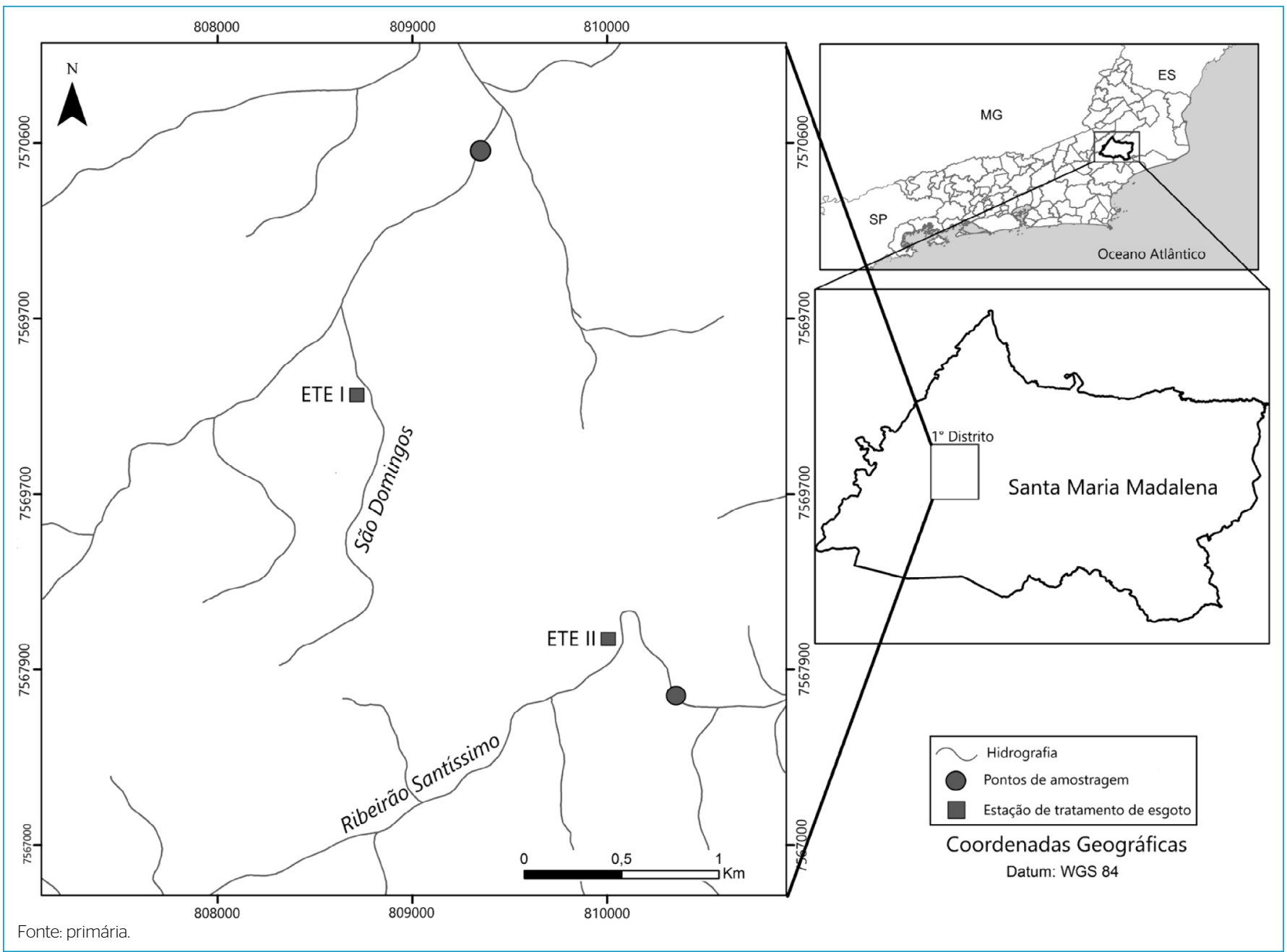

Figura 2 - Localização da área de estudo: município de Santa Maria Madalena (RJ). 
programados, durante um dia inteiro. Esse tipo de amostragem contribui para minimizar os efeitos da variabilidade das concentrações ao longo do dia, dando uma ideia da concentração média (VON SPERLING, 2012).

No campo, as amostras foram coletadas com a ajuda de uma garrafa de $2 \mathrm{~L}$ de Van Dorn (AFK 34) e transferidas parceladamente para garrafas de vidro âmbar de $1 \mathrm{~L}$, em duplicata, que ao fim do dia de coleta estavam cheias. Para impedir a biodegradação dos analitos de interesse, foram adicionados $10 \mathrm{~mL}$ de metanol a cada $1 \mathrm{~L}$ de amostra. Em seguida, as amostras foram levadas para o Laboratório de Toxicologia do Centro de Estudos da Saúde do Trabalhador e Ecologia Humana (CESTEH/FIOCRUZ). Em todo o processo de amostragem e percurso até o laboratório, as amostras foram mantidas em caixas de isopor com gelo e depois armazenadas a $4^{\circ} \mathrm{C}$.

\section{Reagentes}

Preparou-se uma solução padrão de E2 (98\% de pureza, Sigma-Aldrich ${ }^{\oplus}$ ) em acetona, que foi armazenada a $4^{\circ} \mathrm{C}$. Todos os reagentes foram obtidos da Sigma-Aldrich ${ }^{\circledast}$, exceto biotina e CPRG, fornecidos pela Merck $^{\circledast}$. A água purificada foi obtida com o uso de um sistema Milli-Q Biocell (Millipore ${ }^{\circledR}$ ).

\section{Preparação das amostras para ensaio de atividade estrogênica}

No laboratório, as amostras foram filtradas em até 48 horas após a coleta, com o auxílio de uma bomba a vácuo. Primeiramente, utilizou-se uma membrana de celulose de 0,45 $\mu \mathrm{m}\left(\right.$ Merck $\left.^{\circledast}\right)$ e depois um filtro de fibra de vidro de 0,22 $\mu \mathrm{m}\left(\mathrm{MFS}^{\circledast}\right)$. Na sequência, o $\mathrm{pH}$ das amostras foi ajustado para 2 com a adição de uma solução de ácido clorídrico ( 3 mol.:-1 $)$.

Ainda com o auxílio de uma bomba de vácuo e um manifold (Waters ${ }^{\circledR}$ ), efetuou-se o procedimento de extração em fase sólida (EFS). Os cartuchos Oasis HLB $\left(\right.$ Waters $^{\circledR}$ ) foram condicionados sequencialmente com $5 \mathrm{~mL}$ de acetato de etila, $5 \mathrm{~mL}$ de metanol e $5 \mathrm{~mL}$ de água ultrapura, com uma vazão de 5 a $10 \mathrm{~mL}$. $\mathrm{min}^{-1}$. Logo depois, $500 \mathrm{~mL}$ da amostra foram passados pelo cartucho com a mesma vazão. Em seguida, os cartuchos foram submetidos a uma limpeza (clean $u p$ ), com a percolação de $2 \times 5 \mathrm{~mL}$ de água ultrapura com $\mathrm{pH} 2$, e foram secos por aspiração a vácuo durante 10 minutos.

A eluição dos analitos retidos nos cartuchos EFS foi realizada utilizando $4 \mathrm{~mL}$ de acetona. Os eluatos foram então recolhidos em frascos âmbar de $20 \mathrm{~mL}$, evaporados sob uma corrente de nitrogênio e reconstituídos com $2 \mathrm{~mL}$ de etanol.

Para a realização do ensaio YES, as amostras reconstituídas foram levadas para o Laboratório de Engenharia Sanitária (LES/UERJ) em caixas de isopor, sob a temperatura de $4^{\circ} \mathrm{C}$.

\section{Atividade estrogênica pelo ensaio yeast estrogen screen}

A metodologia adotada para a determinação da atividade estrogênica pelo ensaio in vitro YES foi baseada em Routledge e Sumpter (1996). O ensaio foi realizado em microplacas de 96 poços de fundo plano e preparado em uma capela de fluxo laminar.

A solução padrão de E2 (54.48 $\left.\mu \mathrm{g} . \mathrm{L}^{-1}\right)$ e os extratos das amostras foram diluídos em série em etanol, e alíquotas de $10 \mu \mathrm{L}$ de cada diluição foram transferidas para cada poço das microplacas (em duplicata), onde logo em seguida foram evaporadas até a secura. Na sequência, foram adicionados aos poços $200 \mu \mathrm{L}$ do meio de análise (meio de cultivo, levedura e CPRG).
As microplacas foram então seladas com fita adesiva e agitadas vigorosamente durante 2 min em um agitador de placas (IKA MS3 ${ }^{\circledR}$ ). Posteriormente, foram mantidas durante 72 horas a $30^{\circ} \mathrm{C}$ numa incubadora (Nova Ética, $410^{\circledR}$ ). Após o período de incubação, a absorvância foi lida a $575 \mathrm{~nm}$ (para cor) e a $620 \mathrm{~nm}$ (para turbidez) com um leitor de placas VersaMax (Molecular Devices ${ }^{\circledast}$ ).

Em cada ensaio, foram utilizados etanol e E2 (diluído em série) como controle negativo e controle positivo, respectivamente. Os valores de absorção das amostras foram corrigidos de acordo com a Equação 1 e os gráficos foram construídos com as curvas dose-resposta do ensaio.

$\mathrm{Abs}_{\text {Amostra corrigida }}=\mathrm{Abs}_{575 \text { Amostra }}-\left(\frac{A b s_{620 \text { Amostra }}}{A b s_{620 \text { Branco }}}\right)$

As curvas dose-resposta para o padrão E2 e para os extratos das amostras foram plotadas com a concentração versus a resposta estrogênica (absorvância corrigida), resultando em curvas sigmoides que foram ajustadas pelo software Origem $6.0\left(\right.$ Microsoft $\left.^{\circledR}\right)$.

Calculou-se a $\mathrm{EC}_{50}$, que é $50 \%$ da maior resposta da $\beta$-galactosidase obtida no ensaio YES, em relação à curva padrão. Neste estudo, as curvas dose-resposta para o controle positivo E2 foram feitas na faixa de concentração de 2,724 a 1,33 ng. $\mathrm{L}^{-1}$, e o limite de detecção (LD) e o limite de quantificação (LQ) foram de $0,02 \pm 0.01$ ng. $L^{-1}$ e $0,09 \pm 0,04$ ng. $L^{-1}$, respectivamente.

Além da determinação da $\mathrm{EC}_{50}$, $\mathrm{EQ}$-E2 também foi calculado com base na indução máxima de $\beta$-galactosidase em cada extrato de amostra, pela interpolação dos dados das curvas das amostras com os da curva de controle positivo E2 (em ng.L-1 ${ }^{-1}$. Para obter o valor real de EQ-E2 da amostra de água superficial (em ng. $\left.\mathrm{L}^{-1}\right)$, foi necessário dividir o valor EQ-E2 (em ng. $\left.\mathrm{L}^{-1}\right)$ pelo fator de concentração usado na EFS.

Durante o ensaio YES, a presença de compostos citotóxicos nas amostras pode inibir o crescimento da levedura Saccharomyces cerevisiae. A inibição do crescimento das células pode ser acompanhada pela redução dos valores de absorvância a $620 \mathrm{~nm}$ em cada poço, e a citotoxicidade pode ser avaliada de acordo com a Equação 2, como descrito por Frische et al. (2009).

Citotoxicidade $=1-\left(\frac{A b s_{620 \text { Amostra }}}{\text { Abs }_{620 \text { Controle Negativo }}}\right)$

\section{Avaliação de risco}

A avaliação de risco baseou-se nos quocientes de risco (QR, ou em inglês risk quotients - RQ) conforme os estudos de Kunz et al. (2015), que se baseou na WFD. O QR é expresso como a relação analítica entre as concentrações ambientais medidas (CAM, ou em inglês measured environmental concentrations - MEC) ou as concentrações do EQ-E2 e o padrão médio da qualidade ambiental (PMQA, ou em inglês annual average environmental quality standard - AA EQS), como mostra a Equação 3:

$\mathrm{QR}=\frac{\mathrm{CAM} \text { ou EQ-E2 }}{\mathrm{PMQA}}$

Segundo o PMQA, a concentração de E2 para proteger o meio aquático em águas superficiais é de 0.4 ng.L.-1 (SCHER, 2011; UNIÃO EUROPEIA, 2013). Para a interpretação dos dados, o risco ambiental varia entre: nenhum $(<1)$, em risco ( 1 a 10$)$ e alto risco (> 10), em que efeitos nocivos podem ser esperados em razão da presença de compostos estrogênicos na água (KUNZ et al., 2015). 


\section{RESULTADOS E DISCUSSÃO}

\section{Atividade estrogênica}

Inicialmente, observou-se que as amostras dos córregos São Domingos e Ribeirão Santíssimo não apresentaram citotoxicidade. Ou seja, as substâncias presentes nas amostras de ambos os corpos hídricos não inibiram o crescimento ou não mataram a levedura Saccharomyces cerevisiae, organismo base para condução do ensaio YES.

Para efeito de validação do E2 enquanto controle positivo e indutor de atividade estrogênica, foi traçada e analisada a curva dose-resposta do E2 (Figura 3). Observou-se que os resultados foram satisfatórios, uma vez que essa curva apresentou formato sigmoide. Além disso, o controle negativo (branco) também foi satisfatório, não apresentando qualquer contaminação.

Com relação à atividade estrogênica das amostras de águas superficiais, as do córrego São Domingos foram as únicas que apresentaram resposta positiva, como mostra a Figura 4. Esse resultado pode estar associado ao fato de a área de

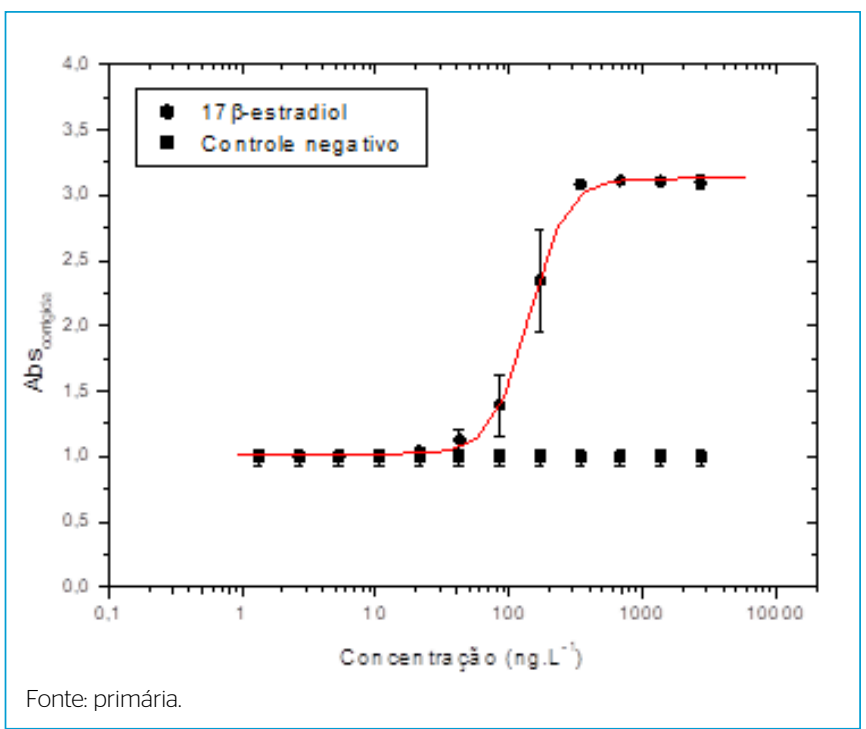

Figura 3 - Curva dose-resposta do controle positivo 17 $\beta$-estradiol no ensaio YES.

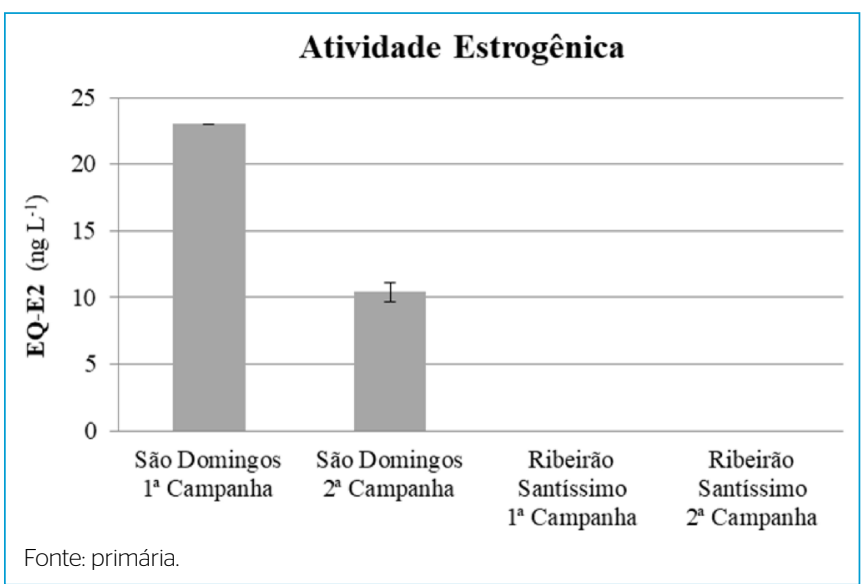

Figura 4 - Atividade estrogênica das amostras de águas superficiais do córrego São Domingos e Ribeirão Santíssimo, Santa Maria Madalena (RJ). contribuição desse corpo hídrico ser mais adensada, abrigando o centro da cidade de Santa Maria Madalena e uma série de outros bairros. Além disso, quando comparada com o córrego Ribeirão Santíssimo, a área drenante ao córrego São Domingos conta com menor cobertura de esgotamento sanitário - apenas um de seus quatro bairros é atendido por sistema de coleta e tratamento de esgotos.

Estudos corroboram esta associação: atividade estrogênica e lançamento de efluentes domésticos sem tratamento. Isso porque o E2 e EE2 são os principais contribuintes para os efeitos estrogênicos no meio hídrico, pois são excretados pelas fezes e urina através de seus metabólitos (TING; PRAVEENA, 2017; LV et al., 2016; OMORUYI; POHJANVIRTA, 2015; ZHAO et al., 2015; MANICKUM; JOHN, 2014; BALSIGER et al., 2010). Ademais, alguns trabalhos na literatura mostram a ocorrência de estrogênios na sua forma livre em esgoto bruto e efluentes de ETE, o que se explica por um processo natural no qual as bactérias presentes no meio (por exemplo, Escherichia coli) são capazes de reativar esses metabólitos estrogênicos em sua forma livre (CUNHA et al., 2017; ESTEBAN et al., 2014; FENT; WESTON; CAMINADA, 2006; D'ASCENZO et al., 2003). A concentração média de EQ-E2 encontrada no córrego São Domingos foi de 23 e 10,4 ng.L-1 para agosto e novembro de 2014, respectivamente. Esse resultado mostra a presença de fatores sazonais, uma vez que, no período de estiagem ( $1^{\text {a }}$ campanha), o córrego São Domingos apresentava baixa vazão, associada à menor capacidade de diluição, se comparada ao início do período chuvoso (2a campanha).

Esse comportamento também foi observado em outros estudos que envolvem a determinação de microcontaminantes em águas superficiais. Montagner e Jardim (2011) analisaram as variações espaciais e sazonais de produtos farmacêuticos e desreguladores endócrinos no rio Atibaia, São Paulo, Brasil, e observaram que no período de estiagem foram obtidas concentrações mais altas dessas substâncias. A concentração máxima de E2, por exemplo, durante o período de maior precipitação, foi de $6,8 \mathrm{ng} . \mathrm{L}^{-1}$, enquanto o valor de $106 \mathrm{ng} . \mathrm{L}^{-1}$ foi determinado no período da seca.

Comparando com a literatura que documenta estudos que também adotaram o YES como bioensaio, Osman et al. (2015) investigaram a atividade estrogênica no rio Nilo, no Egito, e encontraram concentrações de EQ-E2 em níveis mais baixos quando comparados com os do presente estudo, sendo a menor concentração de 0.036 ng. $\mathrm{L}^{-1}$ e a maior de 0,042 ng. $\mathrm{L}^{-1}$. No rio Ebro, na Espanha, também se observou baixo valor de EQ-E2 em águas superficiais, de 2 ng.L-1 (GORGA et al., 2015). Mais recentemente, verificou-se a estrogenicidade em diversos rios da China, e observaram-se baixas e altas concentrações, com valores que variaram de 0,01 a 40,27 ng. $\mathrm{L}^{-1} \mathrm{em}$ águas superficiais (YAO et al., 2018). E no Uruguai, segundo o estudo realizado por Griffero et al. (2018) na matriz aquática do rio Santa Lucía, observou-se um EQ-E2 de 2,4 ng.L-1 em apenas um dos três pontos analisados, no mês de janeiro.

No Brasil, mais especificamente no estado do Rio de Janeiro, um estudo realizado por Dias et al. (2015) analisou a atividade estrogênica no rio Paraíba do Sul e encontrou valores de EQ-E2 de 0,9, 1,7 e 3,1 ng.L L $^{-1}$ nas regiões dos municípios de Resende, Barra Mansa e Volta Redonda, respectivamente. Esse resultado corrobora os do presente estudo, uma vez que a concentração de EQ-E2 aumentou para locais a montante e a jusante do rio, mostrando que a contribuição de lançamentos de esgoto é um fator importante com relação à estrogenicidade.

\section{Avaliação de risco}

O córrego Ribeirão Santíssimo não apresentou risco, uma vez que o QR foi $<1$, valor já esperado, pois não foi observado resultado positivo do EQ-E2 no 
ensaio YES. Ou seja, de acordo com a Diretiva 2013/39/EU, esse corpo hídrico está em conformidade com os padrões de qualidade ambiental para substâncias estrogênicas (KUNZ et al., 2015; UNIÃO EUROPEIA, 2013; SCHER, 2011). Entretanto, o córrego São Domingos apresentou alto risco, com QR > 10, sendo 57,5 para agosto e 26,05 para novembro. Segundo a literatura, esses resultados devem ser considerados ecologicamente relevantes pela possibilidade de ocorrerem graves efeitos adversos em espécies aquáticas.

O cálculo adotado pela Comissão Europeia para estipular o nível de efeito adverso não observado do E2 levou em consideração um estudo realizado por Lahnsteiner et al. (2006), que propôs o limite de 0,4 ng.L $\mathrm{L}^{-1}$ para a concentração do $\mathrm{E} 2$ nos corpos hídricos. Esse estudo indica ainda que concentrações de E2 $\geq 1 \mathrm{ng} . \mathrm{L}^{-1}$ podem reduzir severamente a capacidade reprodutiva de peixes, por causa da combinação negativa da diminuição do volume de sêmen, densidade, motilidade e fertilidade espermática (SCHER, 2011).

\section{CONCLUSÕES}

O ensaio YES forneceu bons resultados sobre a atividade estrogênica em amostras de águas doces superficiais com contribuição de efluentes domésticos. Além disso, confirma-se que a utilização desse ensaio pode ser de grande valia na detecção da presença de desreguladores endócrinos com atividade estrogênica e na estimativa de seus potenciais efeitos, sendo um importante indicativo de possíveis riscos à biota aquática.

Sobre os resultados obtidos nesta investigação, cabe ressaltar que apenas apresentaram atividade estrogênica as amostras do córrego São Domingos, que apresenta maior aporte de esgoto in natura. Observou-se, ainda, maior atividade estrogênica no período de estiagem, quando é menor a vazão do corpo hídrico e, consequentemente, menor sua capacidade de diluição de poluentes.

O Brasil vem seguindo o caminho da expansão de estudos sobre essa temática. No entanto, ainda são observadas lacunas significativas no conhecimento sobre a presença e os efeitos dessa nova classe de contaminantes nos ambientes em que são liberados. Como se trata de um tema relativamente novo, a investigação e o monitoramento da presença de desreguladores endócrinos nos corpos hídricos contribuem para a maior compreensão dos potenciais impactos desses contaminantes emergentes e, ainda, para a elaboração de regulamentos e medidas de controle (CUNHA et al., 2016).

\section{CONTRIBUIÇÕES DOS AUTORES}

Cunha, D. L.: Conceituação, Curadoria de Dados, Análise Formal, Investigação, Metodologia, Administração do Projeto, Validação, Escrita - Primeira Redação. Muylaert, S.: Curadoria de Dados, Análise Formal, Investigação, Metodologia, Supervisão, Escrita - Primeira Redação. Nascimento, M. T. L.: Metodologia, Visualização. Silva, G. G. M.: Curadoria de Dados, Metodologia, Visualização, Escrita - Revisão e Edição. Fonseca, E. F.: Análise Formal, Escrita - Revisão e Edição. Bila, D. M.: Análise Formal, Metodologia, Recursos, Supervisão, Escrita - Revisão e Edição. Sarcinelli, P. N.: Análise Formal, Metodologia, Recursos, Supervisão, Visualização, Escrita - Revisão e Edição. Larentis, A. L.: Análise Formal, Metodologia, Recursos, Supervisão, Visualização, Escrita Revisão e Edição.

\section{REFERÊNCIAS}

ABDEL-KHALEK, A.A. Chronic exposure to water of lake qaroun induced metal-related testicular damage and endocrine disruption in male fish. Biological Trace Element Research, v. 185, n. 1, p. 197-204, 2018. https://doi. org/10.1007/s12011-017-1220-y

ALVAREZ, D.A.; SHAPPELL, N.W.; BILLEY, L.O.; BERMUDEZ, D.S.; WILSON, V.S.; KOLPIN, D.W.; PERKINS, S.D.; EVANS, N.; FOREMAN, W.T; GRAY, J.L.; SHIPITALO, M.J.; MEYER, M.T. Bioassay of estrogenicity and chemical analyses of estrogens in streams across the United States associated with livestock operations. Water Research, v. 47, n. 10, p. 3347-3363, 2013. https:// doi.org/10.1016/j.watres.2013.03.028

AQUINO, S.F.; BRANDT, E.M.F.; CHERNICHARO, C.A.L. Remoção de fármacos e desreguladores endócrinos em estações de tratamento de esgoto: revisão da literatura. Engenharia Sanitária e Ambiental, v. 18, n. 3, p. 187-204, 2013. https://doi.org/10.1590/S1413-41522013000300002

BALSIGER, H.A.; DE LA TORRE, R.; LEE, W.Y.; COX, M.B. A Four-Hour Yeast Bioassay for the Direct Measure of Estrogenic Activity in Wastewater without Sample Extraction, Concentration, or Sterilization. Science of the Total Environment, v. 408, n. 6, p. 1422-1429, 2010. https://doi.org/10.1016\%2Fj. scitotenv.2009.12.027

BRANDT, E.M.F.; QUEIROZ, F.B.; AFONSO, R.J.C.F.; AQUINO, S.F.; CHERNICHARO, C.A.L. Behaviour of pharmaceuticals and endocrine disrupting chemicals in simplified sewage treatment systems. Journal of Environmenta/Management, v. 128, p. 718-726, 2013. https://doi.org/10.1016/j.jenvman.2013.06.003

BRIX, R.; NOGUEROL, T.N.; PIÑA, B.; BALAAM, J.; NILSEN, A.J.; TOLLEFSEN K.E.; LEVY, W.; SCHRAMM, K.W.; BARCELO, D. Evaluation of the suitability of recombinant yeast-based estrogenicity assays as a pre-screening tool in environmental samples. Environment International, v. 36, n. 4, p. 361-367, 2010. https://doi.org/10.1016/j.envint.2010.02.004

CHORMEY, D.S.; BÜYÜKPINAR, Ç: TURAK, F; KOMESLI, O.T; BAKIRDERE, S. Simultaneous determination of selected hormones, endocrine disruptor compounds, and pesticides in water medium at trace levels by GC-MS after dispersive liquid-liquid microextraction. Environmental Monitoring and Assessment, v. 189, n. 6, p. 277-287, 2017. https://doi.org/10.1007/s10661-017-6003-6

CUNHA, D.L.; PAULA, L.M.; SILVA, S.M.C.; BILA, D.M.; FONSECA, E.M.; OLIVEIRA, J.L. Ocorrência e remoção de estrogênios por processos de tratamento biológico de esgotos. Revista Ambiente \& Água, v. 12, n. 2, p. 249-262, 2017. http://doi.org/10.4136/ambi-agua.1992

CUNHA, D.L.; SILVA, S.M.C.; BILA, D.M.; OLIVEIRA, J.L.; SARCINELLI, P.N LARENTIS, A.L. Regulamentação do estrogênio sintético 17 $\alpha$-etinilestradiol em matrizes aquáticas na Europa, Estados Unidos e Brasil. Cadernos de Saúde Pública, v. 32, n. 3, e00056715, 2016. https://doi.org/10.1590/0102$311 \times 00056715$ 
D’ASCENZO, G.; DI CORCIA, A.; GENTILI, A.; MANCINI, R.; MASTROPASQUA, R.; NAZZARI, M.; SAMPERI, R. Fate of natural estrogen conjugates in municipal sewage transport and treatment facilities. Science of the Total Environment, v. 302, n. 1-3, p. 199-209, 2003. https://doi.org/10.1016/s00489697(O2)00342-x

DIAS, C.V.; GOMES, F.W.; BILA, D.M.; SANT'ANNA JR., G.L.; DEZOTTI, M. Analysis of estrogenic activity in environmental waters in Rio de Janeiro state (Brazil) using the yeast estrogen screen. Ecotoxicology and Environmental Safety, v. 120, p. 41-47, 2015. https://doi.org/10.1016/j.ecoenv.2015.05.013

DVOŘÁKOVÁ, M.; KEJLOVÁ, K.; BENDOVÁ, H.; RUCKI, M.; KOHOUT, P.; VAVROUŠ, A.; JÍROVÁ, D. Alternative methods in vitro for screening of endocrine disruptors. Neuro Endocrinology Letters, v. 37, supl. 1, p. 123-131, 2016.

ESTEBAN, S.; GORGA, M.; PETROVIC, M.; GONZÁLEZ-ALONSO, S.; BARCELO, D.; VALCARCEL, Y. Analysis and occurrence of endocrine-disrupting compounds and estrogenic activity in the surface waters of Central Spain. Science of the Total Environment, v. 466-467, p. 939-951, 2014. https://doi. org/10.1016/j.scitotenv.2013.07.101

FANG, T.Y; PRAVEENA, S.M.; DEBURBURE, C.; ARIS, A.Z.; ISMAIL, S.N.S.; RASDI, I. Analytical techniques for steroid estrogens in water samples - A review. Chemosphere, v. 165, p. 358-368, 2016. https://doi.org/10.1016/j. chemosphere.2016.09.051

FENT, K.; WESTON, A.A.; CAMINADA, D. Ecotoxicology of human pharmaceuticals. Aquatic Toxicology, v. 76, n. 2, p. 122-159, 2006. https://doi. org/10.1016/j.aquatox.2005.09.009

FERRERA, Z.S.; SANTANA, C.M.; RODRÍGUEZ, J.J.S. Analytical methodologies for the determination of endocrine disrupting compounds in biological and environmental samples. BioMed Research International, v. 2013, 674838, 2013. https://doi.org/10.1155/2013/674838

FRISCHE, T.; FAUST, M.; MEYER, W.; BACKHAUS, T. Toxic masking and synergistic modulation of the estrogenic activity of chemical mixtures in a yeast estrogen screen (YES). Environmental Science and Pollution Research, v. 16, n. 5, p. 593-603, 2009. https://doi.org/10.1007/s11356-009-0184-7

GE, J.; CONG, J.; SUN, Y.; LI, G.; ZHOU, Z:; QIAN, C.; LIU, F. Determination of endocrine disrupting chemicals in surface water and industrial wastewater from Beijing, China. Bulletin of Environmental Contamination and Toxicology, v. 84, n. 4, p. 401-405, 2010. https://doi.org/10.1007/s00128-010-9958-3

GILBERT, N. Drug-pollution law all washed up. Nature, v. 491, n. 7425, p. 503504, 2012. https://doi.org/10.1038/491503a

GLINEUR, A.; BARBERA, B.; NOTT, K.; CARBONNELLE, P.; RONKART, S.; LOGNAY, G.; TYTECA, E. Trace analysis of estrogenic compounds in surface and groundwater by ultra-high performance liquid chromatographytandem mass spectrometry as pyridine-3-sulfonyl derivatives. Journal of Chromatography A, v. 1534, p. 43-54, 2018. https://doi.org/10.1016/j. chroma.2017.12.042

GORGA, M.; INSA, S.; PETROVIC, M.; BARCELO', D. Occurrence and spatial distribution of EDCs and related compounds in waters and sediments of Iberian rivers. Science of the Total Environment, v. 503-504, p. 69-86, 2015. https://doi.org/10.1016/.scitotenv.2014.06.037

GRIFFERO, L.; GOMES, G.; BERAZATEGUI, M.; FOSALBA, C.; TEIXEIRA DE MELLO, F.; REZENDE, C.E.; BILA, D.M.; GARCIAA-ALONSO, J. Estrogenicity and cytotoxicity of sediments and water from the drinkwater source- basin of Montevideo city, Uruguay. Ecotoxicology and Environmental Contamination, v. 13, n. 1, p. 15-22, 2018. https://doi.org/10.5132/eec.2018.01.02

JOINT RESEARCH CENTRE INSTITUTE FOR ENVIRONMENT AND SUSTAINABILITY (JRC). Development of the first watch list under the environmental quality standards directive. Ispra: European Commission, 2015. Disponivel em: http://publications.jrc.ec.europa.eu/repository/ bitstream/JRC95018/Ibna27142enn.pdf. Acesso em: 2 fev. 2021.

KUNZ, P.Y; KIENLE, C.; CARERE, M.; HOMAZAVA, N.; KASE, R. In vitro bioassays to screen for endocrine active pharmaceuticals in surface and waste waters. Journal of Pharmaceutical and Biomedical Analysis, v. 106, p. 107-115, 2015. https://doi.org/10.1016/j.jpba.2014.11.018

KUNZ, P.Y.; SIMON, E.; CREUSOT, N.; JAYASINGHE, B.S.; KIENLE, C.; MALETZ, S.; SCHIFFERLI, A.; SCHÖNLAU, C.; AIIT-AIISSA, S.; DENSLOW, N.D.; HOLLERT, H.; WERNER, l.; VERMEIRSSEN, E.L.M. Effect-based tools for monitoring estrogenic mixtures: Evaluation of five in vitro bioassays. Water Research, $\mathrm{v}$ 110, p. 378-388, 2017. https://doi.org/10.1016/j.watres.2016.10.062

LAHNSTEINER, F; BERGER, B.; KLETZL, M.; WEISMANN, T. Effect of $17 \beta$-estradiol on gamete quality and maturation in two salmonid species. Aquatic Toxicology, v. 79, n. 2, p. 124-131, 2006. https://doi.org/10.1016/j. aquatox.2006.05.011

LI, Y.Y; CHEN, J.; QIN, Z.F. Determining the optimal developmental stages of Xenopus laevis for initiating exposures to chemicals for sensitively detecting their feminizing effects on gonadal differentiation. Aquatic Toxicology, v. 179, p. 134-142, 2016. https://doi.org/10.1016/j.aquatox.2016.09.002

LIU, G.; KE, M.; FAN, X.; ZHANG, M.; ZHU, Y.; LU, T.; SUN, L.; QIAN, H. Reproductive and endocrine-disrupting toxicity of Microcystis aeruginosa in female zebrafish. Chemosphere, v. 192, p. 289-296, 2018. https://doi. org/10.1016/j.chemosphere.2017.10.167

LV, X.; XIAO, S.; ZHANG, G.; JIANG, P.; TANG, F. Occurrence and removal of phenolic endocrine disrupting chemicals in the water treatment processes. Scientific Reports, v. 6, n. 1, p. 1-10, 2016. https://doi.org/10.1038/srep22860

MANICKUM, T.; JOHN, W. Occurrence, fate and environmental risk assessment of endocrine disrupting compounds at the wastewater treatment works in Pietermaritzburg (South Africa). Science of the Total Environment, v. 468-469, p. 584-597, 2014. https://doi.org/10.1016/j.scitotenv.2013.08.041

MONTAGNER, C.C.; JARDIM, W.F. Spatial and seasonal variations of pharmaceuticals and endocrine disruptors in the Atibaia River, São Paulo State (Brazil). Journal of the Brazilian Chemical Society, v. 22, n. 8, p. 14521462, 2011. https://doi.org/10.1590/S0103-50532011000800008

NEGINTAJI, A.; SAFAHIEH, A.; ZOLGHARNEIN, H.; MATROODI, S. Shortterm induction of vitellogenesis in the immature male yellowfin seabream (Acanthopagrus latus) exposed to bisphenol A and $17 \beta$-estradiol Toxicology and Industrial Health, v. 34, n. 2, p. 119-127, 2018. https://doi. org/10.1177/0748233717748099

NIE, M.; YAN, C.; DONG, W.: LIU, M.; ZHOU, J.; YANG, Y. Occurrence, distribution and risk assessment of estrogens in surface water, suspended particulate matter, and sediments of the Yangtze Estuary. Chemosphere, v. 127, p. 109116, 2015. https://doi.org/10.1016/j.chemosphere.2015.01.021

OMORUYI, I.M.; POHJANVIRTA, R. Estrogenic activity of wastewater, bottled waters and tap water in Finland as assessed by a yeast bio-reporter assay. Scandinavian Journal of Public Health, v. 43, n. 7, p. 770-775, 2015. https://doi. org/10.1177/1403494815591686 
OSMAN, A.G.M.; FADL, K.Y.A.; KRÜGER, A.; KLOAS, W. Screening of multiple hormonal activities in water and sediment from the river Nile, Egypt, using in vitro bioassay and gonadal histology. Environmental Monitoring and Assessment, v. 187, n. 6. p. 317, 2015. https://doi.org/10.1007/s10661-015-4553-z

PLANO MUNICIPAL DE SANEAMENTO BÁSICO DE SANTA MARIA MADALENA (PMSB). Plano regional de saneamento com base municipalizada nas modalidades água, esgoto e drenagem urbana. Londrina: DRZ Gestão Ambiental, 2012. 55 p.

PORSERYD, T.; KELLNER, M.; REYHANIAN CASPILLO, N.; VOLKOVA, K.; ELABBAS, L.; ULLAH, S.; OLSÉN, H.; DINNÉTZ, P.; PORSCH HÄLLSTRÖM, I. Combinatory effects of low concentrations of $17 \alpha$-etinylestradiol and citalopram on non-reproductive behavior in adult zebrafish (Danio rerio). Aquatic Toxicology, v. 193, p. 9-17, 2017. https://doi.org/10.1016/j. aquatox.2017.10.001

ROUTLEDGE, E.J.; SUMPTER, J.P. Estrogenic activity of surfactants and some of their degradation products assessed using a recombinant yeast screen. Environmental Toxicology and Chemistry, v. 15, n. 3, p. 241-248, 1996. https://doi.org/10.1002/etc.5620150303

SCIENTIFIC COMMITTEE ON HEALTH AND ENVIRONMENTAL RISKS (SCHER). Environmental Quality Standards - 17ß-estradiol (E2). SCHER, 2021. Disponível em: https://ec.europa.eu/health/scientific_committees/ environmental_risks/docs/scher_o_13.pdf. Acesso em: 2 fev. 2021.

TAMSCHICK, S.; ROZENBLUT-KOŚCISTY, B.; OGIELSKA, M.; LEHMANN, A.; LYMBERAKIS, P.; HOFFMANN, F.; LUTZ, I.; SCHNEIDER, R.J.; KLOAS, W.; STÖCK, M. Impaired gonadal and somatic development corroborate vulnerability differences to the synthetic estrogen ethinylestradiol among deeply diverged anuran lineages. Aquatic Toxicology, v. 177, p. 503-514, 2016. https://doi.org/10.1016/j.aquatox.2016.07.001

TING, Y.F.; PRAVEENA, S.M. Sources, mechanisms, and fate of steroid estrogens in wastewater treatment plants: a mini review. Environmental Monitoring and Assessment, v. 189, n. 4, p. 178, 2017. https://doi.org/10.1007/ s10661-017-5890-x
UNIÃO EUROPEIA. Diretiva 2013/39/EU - Parlamento Europeu e do Conselho, de 12 de agosto de 2013, que altera as Diretivas 2000/60/CE e 2008/105/CE no que respeita às substâncias prioritárias no domínio da política daágua. Jornal Oficial da União Europeia, 2013. Disponível em: https:// eur-lex.europa.eu/legal-content/ES/ALL/?uri=CELEX\%3A32013L0039. Acesso em: 15 fev. 2021.

VALCARCE, D.G.; VUELTA, E.; ROBLES, V.; HERRÁEZ, M.P. Paternal exposure to environmental 17-alphaethinylestradiol concentrations modifies testicular transcription, affecting the sperm transcript content and the offspring performance in zebrafish. Aquatic Toxicology, v. 193, p. 18-29, 2017 https://doi.org/10.1016/j.aquatox.2017.09.025

VON SPERLING, V.M. Estudos e modelagem da qualidade da água de rios Princípios do Tratamento Biológico de Águas Residuárias. Belo Horizonte: Ed. UFMG, 2012. 588 p.

WEN, Z.; HUANG, X.; GAO, D.; LIU, G.; FANG, C.; SHANG, Y.; DU, J.; ZHAO, Y.; LV L.; SONG, K. Phthalate esters in surface water of Songhua River watershed associated with land use types, Northeast China. Environmental Science and Pollution Research, v. 25, n. 8, p. 7688-7698, 2018. https://doi.org/10.1007/ s11356-017-1119-3

WU, Q.; LAM, J.C.W.; KWOK, K.Y.; TSUI, M.M.P.; LAM, P.K.S. Occurrence and fate of endogenous steroid hormones, alkylphenol ethoxylates, bispheno A and phthalates in municipal sewage treatment systems. Journal of Environmental Sciences, v. 61, p. 49-58, 2017. https://doi.org/10.1016/j. jes.2017.02.021

YAO, B.; LI, R.; YAN, S.; CHAN, S.A.; SONG, W. Occurrence and estrogenic activity of steroid hormones in Chinese streams: A nationwide study based on a combination of chemical and biological tools. Environmental International, v. 118, p. 1-8, 2018. https://doi.org/10.1016/j.envint.2018.05.026

ZHAO, J.L.; CHEN, X.W.; YAN, B.; WEI, C.; JIANG, Y.X.; YING, G.G. Estrogenic activity and identification of potential xenoestrogens in a coking wastewater treatment plant. Ecotoxicology and Environmental Safety, v. 112, p. 238-246 2015. https://doi.org/10.1016/j.ecoenv.2014.11.012 